\title{
Evaluation of Adherence to Chemotherapy-Induced Nausea and Vomiting Guidelines. An Observational Study*
}

\author{
Saja Almazrou, Lamya Alnaim
}

King Saud University, College of Pharmacy, Riyadh, Saudi Arabia.

Email: Salmazrou@ksu.edu.sa, lalnaim@ksu.edu.sa

Received August $17^{\text {th }}, 2012$; revised September $20^{\text {th }}$, 2012; accepted September $30^{\text {th }}, 2012$

\begin{abstract}
Objective: To describe the prescribing trends of antiemetics in chemotherapy-induced nausea and vomiting (CINV), assess adherence to American Society of Clinical Oncology (ASCO) guidelines, and evaluate the effectiveness of prescribed antiemetics. Additionally, we also sought to explore barriers that hinder clinical practice guideline (CPG) implementation. Methods: One hundred fifty-five patients between the ages of 18 and 60 who were admitted to the haematology/oncology department/clinic to receive intravenous chemotherapy, either as in-patients or outpatients, were enrolled in a prospective observational study. Relevant patient demographic data, chemotherapy protocols and antiemetics were collected. Chemotherapies were classified according to their emetogenic potential. This information was used to assess whether the antiemetic prescribed matched the emetogenic risk of treatment. The analysis of outcomes was performed using the MASCC antiemetic assessment tool. Key Findings: The results showed that 95\% of antiemetic prescription pre-chemotherapy regimens did not adhere to the guidelines. The findings were use of twice the recommended dose of granisetron (87.7\%), overuse granisetron (16\%) and metoclopramide (62.6\%), and underuse dexamethasone (27\%) and corticosteroid duplication (7.7\%). With respect to post-chemotherapy antiemetic prescriptions, $91 \%$ of prescriptions were not adherent to guidelines, with overuse of granisetron (81.9\%) and metoclpramide (34.2\%) and under-use of dexamethasone (66.5\%) being the most frequently reported trends. Conclusion: This study shows a lack of conformity to antiemetic guidelines, resulting mainly in overtreatment. Although vomiting was well-managed, nausea remains under controlled and requires additional medical attention. The lack of knowledge and motivation are considered barriers to CPG implementation.
\end{abstract}

Keywords: Chemotherapy; Nausea; Vomiting; Guidelines

\section{Introduction}

Cytotoxic chemotherapies used in the treatment of different malignancies are known to cause significant side effects. One of the immediate side effects that are most distressing to the patients is nausea and vomiting. Poorly controlled chemotherapy-induced nausea and vomiting (CINV) can lead to dehydration, malnutrition and electrolyte imbalance and can cause physical damage, including Mallory-Weiss tears of the oesophagus. Thesesymptoms can result in treatment delays, or a patient may refuse to continue treatment. CINV can also have an economic impact on the management of patients with cancer, including increased hospitalisation and nursing costs [1].

Three forms of vomiting or nausea induced by cancer chemotherapy can be distinguished: acute emesis arising within $24 \mathrm{~h}$ of chemotherapy administration, delayed emesis arising after the first $24 \mathrm{~h}$ and lasting for up to 6

\footnotetext{
*The Authors declare that they have no conflicts of interest to disclose.
}

days, and anticipatory emesis [2].

Treatment guidelines are useful tools that enable physicians to integrate the latest clinical research into their practices. The large volume of rapidly evolving clinical data related to CINV has been summarised and incorporated into treatment recommendations by well-known and reliable institutions, including MASCC and ASCO. Despite the availability of such guidelines, there is evidence that levels of adherence to and implementations of treatment recommendations are less than optimal.

Although CINV negatively affects the quality of life, it has been found that physicians and nurses markedly underestimated the incidence of delayed nausea and emesis after both high and moderate emetogenic chemotherapy. Delayed nausea and emesis, which may appear even in the absence of acute nausea and emesis, remain important targets for improved therapeutic intervention [3].

How effective is the transfer of antiemetic guidelines in routine clinical practice? In 1998, the Italian Group for Antiemetic research carried out a prospective observa- 
tional study on the use and efficacy of antiemetic drugs. The result was that $80 \%$ of patients were either over- or undertreated, creating unjustified costs and placing the patients at greater risk for emesis [4].

In a further study, Fabi et al. investigated the treatment of delayed-type chemotherapy-induced nausea and vomiting. Only $43 \%$ of patients treated with highly emetogenic cytotoxic, $64 \%$ of patients treated with moderately emetogenic cytotoxicity, and 31\% of the patients treated with low-emetogenic cytotoxic agents received the correct antiemetic treatment for delayed-type vomiting. Moreover, $68 \%$ of the patients received unnecessary antiemetic treatment [5].

Another prospective observational study of chemotherapy-related nausea and vomiting in routine practice was performed in a UK cancer centre by Molassiotis et al. to assess the level of CINV using patient self-reports. Data were collected using the MASCC Antiemetic Tool (MAT), which is an eight-item clinical scale assessing acute and delayed nausea and vomiting after chemotherapy. Their data suggest that while vomiting is well-controlled, nausea remains a significant problem in practice, and optimal management of CINV is yet to be achieved [6].

In 2006, a multicentre, prospective, observational follow-up study, including patients with acute myeloid leukaemia and stem-cell transplant recipients, was performed. Patients recorded emetic episodes and rated their level of nausea daily. Quality of life was assessed through a validated functional living index-emesis questionnaire. A survey of caregivers' predictions of CINV was performed, and the predictions were subsequently compared with the observed CINV. This study concluded that despite $5-\mathrm{HT}_{3}$-antagonist prophylaxis, CINV remains a significant problem in oncohaematology, especially in the delayed phase and in transplant recipients [7].

The conclusion of these studies is that powerful barriers exist between the evidence provided by sound research and clinical practice, and this issue hinders progress toward the optimal use of antiemetic drugs.

Clinical practice guidelines on cancer and cancer supportive care are issued and updated frequently by many well recognized associations and institutions. The American Society of Clinical Oncology is a non-profit organization founded in 1964 with the overarching goals of improving cancer care and prevention. Nearly 30,000 oncology practitioners belong to ASCO, representing all oncology disciplines and subspecialties. Members include physicians and health-care professionals in all levels of the practice of oncology [8]. The Multinational Association of Supportive Care in Cancer (MASCC) is an international, multidisciplinary organization with members representing over sixty (60) countries and five continents. MASCC was founded in 1990 and dedicated to research and education in all aspects of supportive care for patients with cancer, regardless of the stage of their disease [9].

This study aims to describe the prescribing trends of antiemetics in CINV in a tertiary hospital and to assess the level of adherence to ASCO guidelines [8] in order to evaluate the effectiveness of prescribed antiemetics in terms of patient outcomes.

The second objective is to explore barriers that hinder the implementation of clinical practice guidelines (CPG).

\section{Patients and Methods}

A prospective observational study was conducted in the haematology/oncology department in a university hospital over a 3-month period from February to April 2010.

\subsection{Ethical Approval}

The proposal was first submitted to the head of the department of haematology/oncology for approval. Subsequently, it was reviewed in the department's research committee for feedback from physicians treating the potential participants.

The reviewed proposal was submitted to the College of Medicine Research Ethics Committee-King Khalid University Hospital and was approved before any data were collected.

After identifying eligible patients and obtaining the consultant's agreement, verbal consent was obtained from each patient. The verbal consent explained the study aims, objectives and potential value.

All participants were treated with respect from the time they were approached, even if they refused enrolment, as well as throughout their participation. The generated data were anonymised, which ensured confidentiality. Research output data were not traceable to an individual.

\subsection{Sampling}

All consecutive patients between the ages of 18 and 60 admitted to the haematology/oncology department/clinic to receive intravenous chemotherapy as either in-patients or outpatients were eligible for inclusion in the study.

The exclusion criteria included patients receiving radiotherapy, patients receiving oral chemotherapy alone, pregnancy, paediatric patients, and HIV-positive patients, or any condition associated with nausea and vomiting.

\subsection{Data Collection}

Such characteristics as age, gender, diagnosis, stage, cycle number, dose and schedule of chemotherapy, as well as the antiemetic regimen administered, were recorded 
for all patients. Chemotherapies were classified according to their emetogenic potential as high, moderate, low or minimal emetogenic chemotherapies according to the MASCC classification [9].

This classification is necessary for the researchers to decide if the antiemetic prescribed matches the emetogenic risk of the treatment.

Demographic and other clinical data were retrieved from the relevant patient files. All of the data were recorded in a predetermined data collection form to ensure completeness and consistency.

A pilot run with ten cases was carried out to determine if the data collection form was able to capture all the information required for data analysis. All data were collected confidentially without identifying information and will be maintained by the researchers.

Pharmacists and physicians working with these patients were unaware of the study in order to minimise the risk of bias.

The effectiveness and tolerability of antiemetic treatment was evaluated by following up with each patient. The presence of nausea and vomiting that occurred during the first 24 hours after the start of cancer chemotherapy and after 4 days was recorded using the MASCC Antiemetic Tool (MAT) [10].

After completion of the data collection process, a previously validated questionnaire was self-administered to 10 physicians; 6 of them were returned [11].

The aim of the survey was to explore the possible barriers that affect adaptation to clinical practice guidelines.

The first part of the questionnaire consists of physician demographic data, such as age, gender, current position and years of experience. Next, 15 questions followed, addressing the most common barriers and facilitators in the implementation of clinical practice guidelines using a Likert scale design.

Higher scores denote greater agreement with a given statement.

Finally, open-ended questions were asked to explore other barriers and facilitators not addressed in the originnal questionnaire.

\subsection{Data Analysis}

Collected data was coded using the Statistical Package for Social Sciences (SPSS Inc., Chicago, IL) version 17 software. Descriptive statistical analysis was carried out; the results are presented as frequencies and percentages.

\section{Results}

\subsection{Participant and Treatment Characteristics}

The sample consisted of one hundred fifty five cancer patients. The mean age was 49.5 years $( \pm 15.6)$. There was equal representation of both males and females in the sample. The majority of patients received chemotherapy that is classified to have moderate emetogenicity.

Breast and colorectal cancer were the two most common diagnoses. The complete demographic characteristics are shown in Table 1.

\subsection{Pre-Chemotherapy Antiemetic}

When we looked at antiemetic used before chemotherapy administration, the data showed that the majority (95\%) of antiemetic prescriptions did not adhere to the guidelines. The trend of non-adherence involved the use of twice the recommended dose of granisetron in $87.7 \%$ of cases. A dose of $2 \mathrm{mg}$ intravenously, was administered instead of $1 \mathrm{mg}$ intravenously. We also observed an overuse of granisetron in $16 \%$ of cases where chemotherapy with low emetogenicity was administered. A More common occurrence, (62.6\%), was the overuse of metoclopramide across chemotherapy with different emetogenicity. Also there was a trend for the under use of dexamethasone in cases where it was indicated (27\%). Finally corticosteroid duplication was seen in $7.7 \%$ of cases, where dexamethasone was prescribed as an antiemetic in patients already receiving prednisolone-containing chemotherapy.

\subsection{Post-Chemotherapy Antiemetic}

When we looked at antiemetic used after chemotherapy administration, the data showed that the majority (91\%) of antiemetic prescriptions were not adherent to guidelines. Overuse of granisetron was observed in $81.9 \%$ of prescriptions, underuse of dexamethasone was observed in $66.5 \%$, and overuse of metoclpramide was observed in $34.2 \%$ of prescriptions.

\subsection{The Assessment of Nausea and Vomiting}

Ninety patients were included in the analysis of outcome; the remaining patients were considered lost to follow-up

Table 1. Demographic characteristics of the sample.

\begin{tabular}{lc}
\hline \multicolumn{2}{l}{ Patients characteristics } \\
\hline Total patients (No) & 155 \\
Age, mean \pm SD & $49.5 \pm 15.6$ \\
Gender, No. (\%) & \\
Male & $76(49.0 \%)$ \\
Female & $79(51.0 \%)$ \\
Chemotherapy emetogenicity, No. (\%) & \\
High & $51(32.9 \%)$ \\
Moderate & $77(49.7 \%)$ \\
Low & $20(12.9 \%)$ \\
Minimal & $5(3.2 \%)$ \\
\hline
\end{tabular}


after two failed attempts to contact them on 4th day postchemotherapy.

The remaining data were assessed only for the adherence to ASCO guidelines in terms of using the appropriate antiemetic regimen for the prevention of acute and delayed CINV.

The adherent and non-adherent groups were compared in terms of achieving complete control, i.e. the absence of vomiting episodes for a period of 24 hours (acute phase) and four days post-chemotherapy (delayed phase). See Figure 1.

In the acute phase, $89 \%$ of patients achieved complete control in the adherent group, while $90.7 \%$ achieved the same control in the non-adherent group.

In the delayed phase, $100 \%$ of patients achieved complete control in the adherent group, while $89 \%$ of patients, achieved the same control in the non-adherent group.

\subsection{Clinical Practice Guideline Implementation}

Lack of knowledge and motivation to read the current guidelines and cooperation with other physicians in implementing guidelines achieved the highest scores for reasons non-adherence. Implementing a penalty system for physicians who did not comply with the guidelines and increasing accessibility were the most common recommended facilitators.

One of the practical barriers was that the guidelines may have been implemented but could not be practiced because some recommended drugs, such as aprepitant, which is proven to be highly effective in the prevention of nausea and vomiting, were not available in the hospital. Such drugs are necessary after highly emetogenic chemotherapy involving such drugs as cisplatin.

\section{Discussion}

Our observational study was conducted to determine the appropriate strategy to prevent chemotherapy-induced nausea and vomiting in the clinical practice setting and yielded evidence of a lack of conformity with therapeutic standards. Overtreatment with/overuse of antiemetic was the predominant practice in our setting.

\subsection{Adherence to Pre-Chemotherapy Antiemetics}

\subsubsection{High-Dose Granisetron}

For the management of acute emesis, granisetron was prescribed in twice the recommended dose. A recent RCT in 2009 aimed to assess the non-inferiority of 1 - 3 mg granisetron (GRN) injection for the treatment of acute CINV. The study concluded that data failed to show the non-inferiority of $1 \mathrm{mg}$ to $3 \mathrm{mg}$ of GRN administered as a single dose. However, the rate of complete protection from nausea and vomiting was similar in the two groups. Given the recommended dosage in the guidelines and the economic need for the reduction of medical care expenses, the prophylactic administration of GRN at $1 \mathrm{mg}$ may be an appropriate alternative treatment for acute CINV [12].

\subsubsection{Overuse/Over-Prescription of Metoclopramide}

Metocolpramide was prescribed in $62.6 \%$ of patients. According to ASCO guidelines, metoclopramide is only indicated if the patient is intolerant to any of the $5-\mathrm{HT}_{3}$ antagonists, dexamethasone or aprepitant.

A prospective, randomised, open study was performed to evaluate the antiemetic efficacy and tolerability of a regimen based on a single oral dose of ondansetron $8 \mathrm{mg}$ in comparison with a metoclopramide-based regimen for the prevention of acute chemotherapy-induced emesis.

Complete control of acute vomiting was obtained in $93 \%$ of patients receiving ondansetron, and in $65 \%$ of those receiving metoclopramide $(\mathrm{p}=0.00003)$. Complete

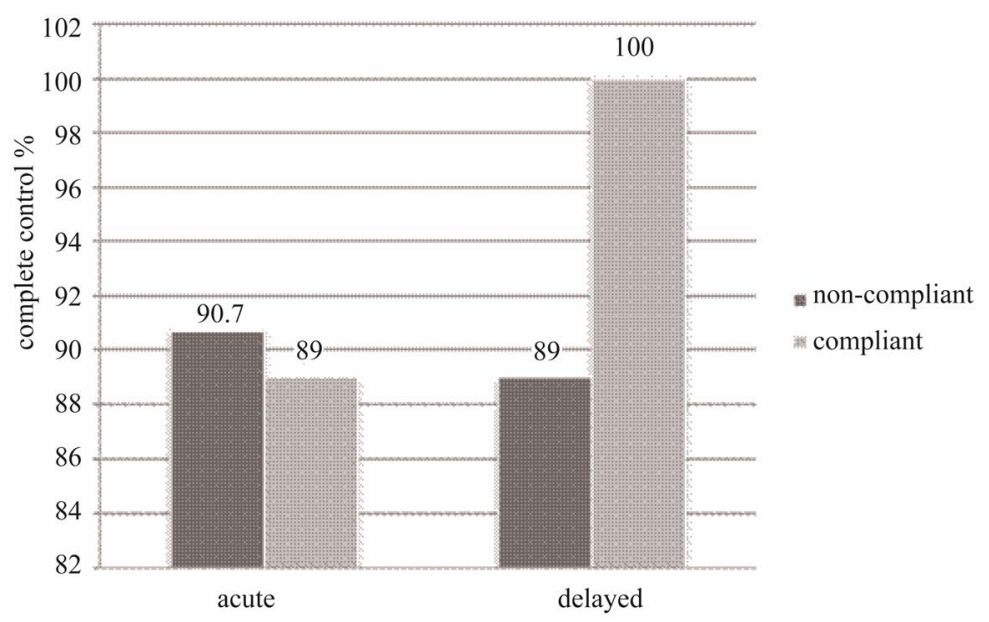

Figure 1. Adherence to ASCO clinical practice. The complete control rates associated with compliant and noncompliant prophylaxis were compared at $24 \mathrm{hr}$ and 4 days after chemotherapy. 
control of acute nausea was obtained in $58 \%$ of patients receiving ondansetron and in $36 \%$ of patients receiving metoclopramide $(p=0.007)$.

The data suggest that ondansetron was highly effective and superior to the metoclopramide-based regimen [13].

A double-blind crossover study sought to compare the antiemetic efficacy and safety of ondansetron, and metoclopramide. In the first 24 hours, complete or major control (zero to two emetic episodes) of emesis was achieved in 30 of 35 (86\%) patients receiving ondansetron and in 14 of 33 (42\%) patients receiving metoclopramide ( $\mathrm{p}<$ 0.001). Ondansetron was also more effective in reducing acute nausea [14].

\subsubsection{Corticosteroid Duplication}

Corticosteroid is widely used in the clinical practice of malignant neoplasms due to its antitumor effect in lymphoproliferative disorders, such as lymphoma, acute lymphoblastic leukaemia, and multiple myeloma. Standard regimens of these haematological disorders include corticosteroid as a chemotherapeutic agent. In these circumstances, corticosteroidis especially effective as an antiemetic agent, anti-inflammatory agent, and anti-allergic drug. However, the use of steroid also has many adverse effects. Careful use of corticosteroid should be considered [15].

In $7.7 \%$ of patients, dexamethasone was prescribed as an antiemetic in patients already receiving prednisolone-containing chemotherapy. This duplication may have a negative impact on patient outcome and increase the tendency of the development of classical corticosteroid side effects aside from associated economic factors.

\subsubsection{Underuse of Dexamethasone}

Dexamethasone was under-prescribed in $27 \%$ of patients. This finding may be attributed to the physician's perception of dexamethasone-associated side effects.

\subsubsection{Overuse of Grainsetron}

Granisetron was prescribed in LEC instead of dexamethasone. This prescription phenomenon occurred in $16 \%$ of cases.

Similar findings were observed by Fabi et al. [5] and Molassiotis et al. [6], who reported overtreatment in LEC and minimal emetogenic chemotherapy with drugs that have collateral side effects that are certainly relevant (stypsis, headache, gastrointestinal disturbances) in patients already being subjected to the administration of toxic drugs, which also indicates unnecessarily high costs.

\subsection{Adherence to Post-Chemotherapy Antiemetics}

\subsubsection{Overuse of Granisetron}

In a randomised double-blind trial comparing the effect of granisetron with that of metoclopramide, both drugs were combined with dexamethasone in the prophylaxis-delayed emesis.

This study concluded that the routine prescription of $5-\mathrm{HT}_{3}$ for delayed phase control is not advisable, as it increases costs without any benefit for the majority of patients. Delayed emesis in the rare patients with acutephase emesis remains an unsolved problem [16].

Another review aimed to investigate the clinical efficacy and drug-acquisition costs associated with administering $5-\mathrm{HT}_{3}$ antagonists beyond 24 hours, as monotherapy or in addition to dexamethasone. The authors found that neither clinical evidence nor considerations of cost effectiveness justify using $5-\mathrm{HT}_{3}$ antagonists beyond 24 hours after chemotherapy for the prevention of delayed emesis [17].

In our setting, grainsetron was prescribed in the vast majority of all patients at discharge (81.9\%), regardless the emetogenicity of the chemotherapy.

\subsubsection{Overuse of Metoclopramide}

Although metoclopramide was associated with more side effects than was dexamethasone, it was found that it was prescribed for delayed emesis in $34.2 \%$ of the cases.

A double-blind, randomised, crossover study was conducted to compare the efficacy and safety of highdose dexamethasone and high-dose metoclopramide in the treatment of chemotherapy-induced nausea and vomiting.

Dexamethasone was found to have less of an adverse effect than metoclopramide on the patient's appetite and activity ( $\mathrm{p}<0.025$ and $\mathrm{p}<0.01$, respectively). Twentyone patients (53\%) developed mild to severe somnolence with metoclopramide compared to only seven patients (18\%) who experienced this adverse effect with dexamethasone ( $p<0.01)$. Six patients $(15 \%)$ developed extrapyramidal manifestations with metoclopramide, but none developed it with dexamethasone.

We conclude that high-dose dexamethasone has greater antiemetic activity and is safer than high-dose metoclopramide [18].

\subsubsection{Underuse of Dexamethasone}

Dexamethasone was under-prescribed in (66.5\%), which may negatively affect patient outcome by not controlling delayed nausea and vomiting.

The underuse of dexamethasone was demonstrated in previous studies. An observational study carried out by Mertens et al. [19] showed that the vast majority of compliance issues involved delayed CINV prevention and the absence of post-chemotherapy corticosteroid administration;only $25 \%$ of cases involved post-chemotherapy steroids, and 52\% were treated with post-chemotherapy $5 \mathrm{HT}_{3}$ receptor antagonists. 
Molassiotis et al. [6] looked into the underuse of dexamethasone. They found that many clinicians are concerned with the use of steroids as antiemetic and often either avoid prescribing steroids, prescribe them for a shorter duration than the recommended one or prescribe lower-than-recommended doses, despite the lack of any evidence of harmful effects from the use of dexamethasone given for 3 - 4 days per chemotherapy cycle.

A study sought to determine the role of dexamethasone in the reduction of cisplatin and etoposide-induced nausea and vomiting in lung cancer patients during and after two chemotherapy cycles. Patients were divided in two groups. Group A consisted of 30 patients who received cisplatin and etoposide with standard antiemetic drugs: ondansetron and metoclopramide. Group B consisted of 30 patients who received the same chemotherapy regimen with the previous antiemetic therapy plus dexamethasone $8 \mathrm{mg}$ intravenously per day during the 3 days of chemotherapy. During and after the 3-day therapy, patients filled in a questionnaire related to the adverse effects of chemotherapy, including nausea and vomiting. There was a significant decrease in the frequency of nausea, as well as acute and delayed vomiting in the group who received antiemetic treatment with ondansetron and metoclopramide plus dexamethasone [18].

The study by Molassiotis et al. [6] is comparable to our study in terms of the number of patients included, the assessment tool and the standard reference guidelines.

The results are shown in Table 2. Despite non-adherence to antiemetic guidelines, the outcome was improved in our setting. This might suggest that the ASCO recommendations do not suit our population, and the overuse of antiemetics is necessary to prevent completely CINV.

Because the majority of prescriptions tended toward the overuse of antiemetics, the cost of antiemetic drugs/ patient was calculated. The non-adhered antiemetic

Table 2. Comparison between the current study findings to A. Molassiotis, et al.[6].

\begin{tabular}{lcc}
\hline Characteristic & $\begin{array}{c}\text { A. Molassiotis, } \\
\text { et al. }\end{array}$ & $\begin{array}{c}\text { Current } \\
\text { study }\end{array}$ \\
\hline Number of patients & 102 & 90 \\
Conformity to guideline in HEC & $41.20 \%$ & $17.80 \%$ \\
Conformity to guideline in MEC & $74.50 \%$ & $8.60 \%$ \\
Conformity to guideline in LEC & $42.90 \%$ & $6.60 \%$ \\
Conformity to guideline in minimal & $66.70 \%$ & $100 \%$ \\
emetogenic chemotherapy & $15.70 \%$ & $9.50 \%$ \\
Vomiting acute & $14.70 \%$ & $8.40 \%$ \\
Delayed & $37.30 \%$ & $37.90 \%$ \\
Nausea acute & $47.10 \%$ & $26.30 \%$ \\
\hline Delayed & & \\
\hline
\end{tabular}

regimen cost three-times the cost of the Adherence to the antiemetic regimen.

\subsection{Causes for Non-Adherence}

In a recent paper published in 2009, the authors investigate the obstacles to the implementation of antiemetic guidelines and reveal that variations in format, goals, physician education, and institutional education may all affect guideline implementation.

Physicians believe that guidelines might improve the quality of care [19-21]. However; the guidelines for implementation may be too rigid. Adherence is more difficult if treatment has not been clearly shown to be highly effective or if the problem addressed is not seen as a critical patient issue.

Finally, individual physicians may not be familiar with specific guidelines [21]. Auditing and feedback, which is obtained using a standard chemotherapy order form, allows physicians to request a standard antiemetic and allow pharmacy services to check chemotherapy orders before dispensing and administration. These modalities seem to be practical for improving the level of adherence to clinical practice guidelines.

This study has certain limitations: the small sample size and unequal distribution of groups made it difficult to assess the correlation between adherence and outcome.

\section{Conclusions}

This study shows that there is a lack of conformity to antiemetic guidelines. The main observation is that there is a trend overtreatment and incurred more economic expenditure. This translated into good patient outcome, but it was not investigated if the same outcome would have been achieved with regular treatment. On the other hand, it might be that the ASCO recommendation does not suit our population, and the overuse of antiemetics is necessary to appropriately prevent CINV.

Future studies should determine the effect of overtreatment in terms of antiemetic-related adverse drug reaction and side effects, and the total cost of care.

In general, vomiting was well-managed, but nausea remains under-controlled. This situation requires additional medical attention and further investigation.

Lack of knowledge and motivation are considered to be most common barriers to CPG implementation in our setting. Auditing and feedback may be the easiest tools to use to encourage adherence to the guidelines [22].

\section{REFERENCES}

[1] D. J. Stewart, "Cancer Therapy, Vomiting, and Antiemetics," Canadian Journal of Physiology and Pharmacology, Vol. 68, No. 2, 1990, pp. 304-313.

doi:10.1139/y90-045 
[2] P. L. Andrews and C. J. Davis, “The Mechanism of Emesis Induced by Anticancer Therapies,” In: P. L. Andrews, Ed., Emesis in Anti-Cancer Therapy, Chapman and Hall, London, 1993, pp. 113-161.

[3] S. M. Grunberg, et al., "Incidence of ChemotherapyInduced Nausea and Emesis after Modern Antiemetics," Cancer, Vol. 100, No. 10, 2004, pp. 2261-2268. doi:10.1002/cncr.20230

[4] The Italian Group for Antiemetic Research, "Transferability to Clinical Practice of the Results of Controlled Clinical Trials: The Case of Antiemetic Prophylactic Treatment for Cancer Chemotherapy-Induced Nausea and Vomiting," Annals of Oncology, Vol. 9, No. 7, 1998, pp. 759-765. doi:10.1023/A:1017132123411

[5] A. Fabi, et al., "Is Delayed Chemotherapy Induced Emesis Well Managed in Oncological Clinical Practice? An Observational Study," Supportive Care in Cancer, Vol. 11, No. 3, 2003, pp. 156-161.

[6] A. Molassiotis, et al., “A Prospective Observational Study of Chemotherapy-Related Nausea and Vomiting in Routine Practice in a UK Cancer Center," Supportive Care in Cancer, Vol. 16, No. 2, 2008, pp. 201-208.

[7] J. López-Jiménez, et al., "Chemotherapy-Induced Nausea and Vomiting in Acute Leukemia and Stem Cell Transplant Patients: Results of a Multicenter, Observational Study,” Haematologica, Vol. 91, No. 1, 2006, pp. 84-91.

[8] M. G. Kris, et al., "American Society of Clinical Oncology Guideline for Antiemetics in Oncology: Update 2006,” Journal of Clinical Oncology, Vol. 24, No. 35, 2006, pp. 2932-2947.doi:10.1200/JCO.2006.06.9591

[9] The Antiemetic Subcommittee of the Multinational Association of Supportive Care in Cancer (MASCC), "Prevention of Chemotherapy- and Radiotherapy-Induced Emesis: Results of the 2004 Perugia International Antiemetic Consensus Conference,” Annals of Oncology, Vol. 17, No. 1, 2006, pp. 20-28.

[10] A. Molassiotis, et al., "Validation and Psychometric Assessment of a Short Clinical Scale to Measure Chemotherapy-Induced Nausea and Vomiting: The MASCC Antiemesis Tool," Journal of Pain and Symptom Management, Vol. 34, No. , 2007, pp. 148-159. doi:10.1016/j.jpainsymman.2006.10.018

[11] M. A. J. Peters, et al., "Room for Improvement? Barriers to and Facilitators for Improvement of Patient Care," Centre for Quality of Care Research (WOK), Radboud University Nijmegen Medical Centre, Nijmegen, 2002.

[12] M. Yonemura, et al., "Randomized Controlled Study Comparing Two Doses of Intravenous Granisetron (1 and $3 \mathrm{mg}$ ) for Acute Chemotherapy-Induced Nausea and Vomiting in Cancer Patients: A Non-Inferiority Trial,” Japanese Journal of Clinical Oncology, Vol. 39, No. 7, 2009, pp. 443-448. doi:10.1093/jjco/hyp036
[13] S. M. Bosnjak, et al., "High Efficacy of a Single Oral Dose of Ondansetron $8 \mathrm{mg}$ versus a Metoclopramide Regimen in the Prevention of Acute Emesis Induced by Fluorouracil, Doxorubicin and Cyclophosphamide (FAC) Chemotherapy for Breast Cancer," Journal of Chemotherapy, Vol. 12, No. 5, 2000, pp. 446-453.

[14] J. Bonneterre, et al., "A Randomized Double-Blind Comparison of Ondansetron and Metoclopramide in the Prophylaxis of Emesis Induced by Cyclophosphamide, Fluorouracil, and Doxorubicin or Epirubicin Chemotherapy,” Journal of Clinical Oncolory, Vol. 8, No. 6, 1990, pp. 1063-1069.

[15] H. Tsurumi, "Steroid Therapy in Oncology," Nihon Rinsho, Vol. 66, No. 1, 2008, pp. 143-147.

[16] M. S. Aapro, et al., “A Randomized Double-Blind Trial to Compare the Clinical Efficacy of Granisetron with Metoclopramide, both Combined with Dexamethasone in the Prophylaxis of Chemotherapy-Induced Delayed Emesis,” Annals of Oncology, Vol. 14, No. 2, 2003, pp. 291297. doi:10.1093/annonc/mdg075

[17] O. Geling and H. G. Eichler, "Should 5-Hydroxytryptamine-3 Receptor Antagonists be Administered beyond 24 Hours after Chemotherapy to Prevent Delayed Emesis? Systematic Re-Evaluation of Clinical Evidence and Drug Cost Implications,” Journal of Clinical Oncology, Vol. 23, No. 6, 2005, pp. 1289-1294. doi:10.1200/JCO.2005.04.022

[18] E. M. Ibrahim, et al., "Antiemetic Efficacy of High-Dose Dexamethasone: Randomized, Double-Blind, Crossover Study with High-Dose Metoclopramide in Patients Receiving Cancer Chemotherapy," European Journal of Cancer \& Clinical Oncology, Vol. 22, No. 3, 1986, pp. 283-288

[19] W. C. Mertens, et al., "Improving the Care of Patients with Regard to Chemotherapy-Induced Nausea and Emesis: The Effect of Feedback to Clinicians on Adherence to Antiemetic Prescribing Guidelines," Journal of Clinical Oncology, Vol. 21, No. 7, 2003, pp. 1373-1378. doi:10.1200/JCO.2003.08.118

[20] T. Sarcev, et al., "The Influence of Dexamethasone in the Decrease of Chemotherapy-Induced Nausea and Vomiting,” Journal of the Balkan Union of Oncology, Vol. 12, No. 2, 2007, pp. 245-252.

[21] S. M. Grunberg, "Obstacles to the Implementation of Antiemetic Guidelines," Journal of the National Comprehensive Cancer Network, Vol. 7, No. 5, 2009, pp. 601605.

[22] P. J. Greco and J. M. Eisenberg, "Changing Physicians' Practices," The New England Journal of Medicine, Vol. 329, Suppl. 1, 1993, pp. 1271-1274. doi:10.1056/NEJM199310213291714 


\section{Abbreviations}

CINV: Chemotherapy-Induced Nausea and Vomiting, ASCO: American Society of Clinical Oncology,
MASCC: Multinational Association of Supportive Care in Cancer, CPG: clinical practice guidelines. 